\title{
The Mitochondrial Protease AFG3L2 Is Essential for Axonal Development
}

\author{
Francesca Maltecca, ${ }^{1 \star}$ Asadollah Aghaie, ${ }^{3 \star}$ David G. Schroeder ${ }^{4}$ Laura Cassina, ${ }^{1}$ Benjamin A. Taylor, ${ }^{4}$ \\ Sandra J. Phillips, ${ }_{4}^{4}$ Mariachiara Malaguti, ${ }^{2}$ Stefano Previtali, ${ }^{2}$ Jean-Louis Guénet, ${ }^{3}$ Angelo Quattrini, ${ }^{2}$ Gregory A. Cox, ${ }^{4}$ \\ and Giorgio Casari ${ }^{1,5}$ \\ ${ }^{1}$ Human Molecular Genetics Unit, and ${ }^{2}$ Neuropathology Unit and Istituto di Neurologia Sperimentale, San Raffaele Scientific Institute, 20132 Milan, Italy, \\ ${ }^{3}$ Departement de Biologie du Developpement, Institut Pasteur, 75724 Paris Cedex 15, France, ${ }^{4}$ The Jackson Laboratory, Bar Harbor, Maine 04609, and \\ ${ }^{5}$ Vita-Salute San Raffaele University, School of Medicine, 20132 Milan, Italy
}

The mitochondrial metalloprotease AFG3L2 assembles with the homologous protein paraplegin to form a supracomplex in charge of the essential protein quality control within mitochondria. Mutations of paraplegin cause a specific axonal degeneration of the upper motoneuron and, therefore, hereditary spastic paraplegia. Here we present two Afg3l2 murine models: a newly developed null and a spontaneous mutant that we found carrier of a missense mutation. Contrasting with the mild and late onset axonal degeneration of paraplegin-deficient mouse, $A f g 3 l 2$ models display a marked impairment of axonal development with delayed myelination and poor axonal radial growth leading to lethality at P16. The increased severity of the Afg 312 mutants is explained by two main molecular features that differentiate AFG3L2 from paraplegin: its higher neuronal expression and its versatile ability to support both hetero-oligomerization and homo-oligomerization. Our data assign to AFG3L2 a crucial role by linking mitochondrial metabolism and axonal development. Moreover, we propose AFG3L2 as an excellent candidate for motoneuron and cerebellar diseases with early onset unknown etiology.

Key words: paraplegin; m-AAA protease; axonal development; mitochondria; neurodegeneration; spinal cord

\section{Introduction}

Mitochondria harbor an evolutionarily conserved proteolytic system mediating the complete degradation of organellar proteins. Central components of this system are the highly conserved ATP-dependent metalloproteases of the AAA-superfamily (ATPases associated with a variety of cellular activities) (Bross et al., 2004). These proteases are known to exert chaperon-like activity, such as the assembly of the respiratory chain complexes, and to participate in protein quality control by binding unfolded peptides and ensuring specificity of proteolysis (Leonhard et al., 1999).

Among these proteases, paraplegin and ATPase family gene 3-like 2 (AFG3L2) form the hetero-oligomeric $m$-AAA protease in the inner mitochondrial membrane. Mutations of paraplegin are responsible for a recessive form of hereditary spastic paraplegia (HSP) (Casari et al., 1998), characterized by axonal degeneration of the longest motor and sensory axons of the CNS. We

Received 0ct. 15, 2007; revised Dec. 21, 2007; accepted Dec. 29, 2007.

This work was supported by Italian Telethon Foundation Grant GGP030310 and European Union Sixth Framework Program Grant FP6-503382. A.A. was recipient of an Association Francaise contre les Myopathies Fellowship. We greatly acknowledge Thomas Langer (Cologne, Germany) and Silvia Nerini Molteni for critical discussion. We thank Giorgia Dina for neuropathology technical assistance.

The authors declare no competing financial interests.

*F.M. and A.A. contributed equally to this work.

Correspondence should be addressed to Dr. Giorgio Casari, Vita-Salute San Raffaele University, Via Olgettina 58 , 20132 Milan, Italy. E-mail: casari.giorgio@hsr.it.

DOI:10.1523/JNEUROSCI.4677-07.2008

Copyright $\odot 2008$ Society for Neuroscience $\quad$ 0270-6474/08/282827-10\$15.00/0 demonstrated that the loss of $m$-AAA complex in HSP primary fibroblasts causes reduced complex I activity and increased sensitivity to oxidant stress, which can both be rescued by exogenous expression of paraplegin. Furthermore, complementation studies in yeast revealed functional conservation of the human $m$-AAA protease (Atorino et al., 2003).

The paraplegin deficient mouse model shows mild and slowly progressive motor impairment associated with distal axonopathy of spinal and peripheral axons, partially recapitulating HSP patients' phenotype. Mitochondrial morphological abnormalities were found in synaptic terminals and in distal regions of axons long before axonal degeneration and correlated with the onset of motor impairment (Ferreirinha et al., 2004).

To gain new insights on the functional role of $m$-AAA protease, in this work we have characterized two mouse models carrying mutations in the same $A f g 3 l 2$ gene. We newly developed a null Afg 312 mutant on FVB strain background and, in addition, we identified the genetic cause, i.e., a single $A f g 3 l 2$ missense mutation, of a previously known spontaneous mutant strain developed on C57BL/6 background. Although these models differ both in the type of mutations and in murine genetic background, they display a strikingly identical phenotype. Indeed, Afg $3 l 2 \mathrm{mu}-$ tants show an extremely severe neuromuscular syndrome beginning at P7 with hindlimbs paraparesis which progresses until complete tetraparesis and death, generally at P16. In contrast with the late onset axon degeneration of paraplegin null model, Afg3l2 mutants show a severe defect in axonal development characterized by delayed myelination and impairment of axonal ra- 
dial growth in both CNS and peripheral nervous system (PNS). Mitochondrial morphology abnormalities are detected in motor and sensory neurons, more frequently in proximity of the nucleus. The enzymatic activities of the respiratory chain complexes are strikingly impaired in Afg3l2 models, thus resulting in highly reduced ATP production.

Previously we have shown that, in addition to the paraplegin-AFG3L2 heterocomplexes, AFG3L2 can also form active homo-oligomers (Koppen et al., 2007). The severe phenotype of AFG3L2deficient mice compared with the paraplegin mutant assign to AFG3L2 a crucial role within $m$-AAA complexes, essential for mitochondrial function in neuronal cells. This is the first report of murine models linking axonal development to a mitochondrial protein. Together, functional and genetic evidences indicate AFG3L2 as an excellent candidate for early onset HSP or neonatal motoneuron diseases with unknown etiology.

\section{Materials and Methods}

Morphological analysis. Morphological analyses were conducted as described previously (Dubowitz, 1985; Previtali et al., 2000). Brain, cerebellum, spinal cord, dorsal root ganglia, muscles, and sciatic nerves were removed, postfixed in $0.12 \mathrm{M}$ phosphate buffer $/ 2 \%$ glutaraldehyde, sectioned into $2 \mathrm{~mm}$ blocks, postfixed with $1 \%$ osmium tetroxide and embedded in Epon (Fluka, Buchs, Switzerland). Semithin sections $(0.5-1 \mu \mathrm{m}$ thick) were stained with toluidine blue and examined by light microscopy (LM; BX51; Olympus, Tokyo, Japan). To perform morphometric analysis, digitalized images of cross sections were obtained from lumbar spinal cord and sciatic nerve with a $100 \times$ objective and a digital camera Leica (Nussloch, Germany) DFC300F. Images were analyzed with the Leica QWin software. Counting of neuronal cell bodies and axons was performed in a blind and unbiased manner on an average of three nonoverlapping microscopic fields per mouse $(n=3)$. Ultrathin sections $(100-120 \mathrm{~nm}$ thick) were stained with uranile acetate and lead citrate and examined by electron microscopy (EM) (magnification $\times 3000$, Leo 912; Omega, Brattleboro, VT). Neurofilament (NF) densities were quantitatively evaluated in mutant and control sciatic nerve fibers by overlaying a transparent square profile onto electron micrographs at a final magnification of $\times 60,000$. Each square represented a $0.25 \mu \mathrm{m}^{2}$ region of the axoplasm, not containing membranebound organelles or Schwann cell infoldings.

Four to six axons in true cross sections were randomly selected for

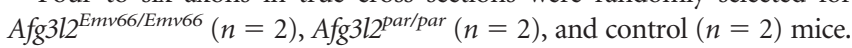
The number of NF present in 2-6 squares/axon was counted. Hematoxylin and eosin staining was done using standard techniques. Modified trichrome staining, determination of cytochrome $c$ oxidase (COX), and succinic dehydrogenase (SDH) activity were performed as described previously (Dubow-

A

C

D ize sample loading.

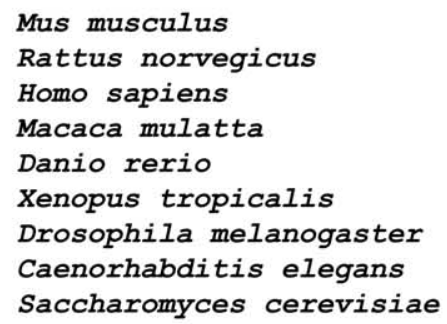

VSGSEFLEMFVGVGPARVRPLFALARKNAPCILFIDE VSGSEFLEMFVGVGPARVRDLFALARKNAPCILFIDE VSGSEFLEMFVGVGPARVRDLFALARKNAPCILFIDE VSGSEFLEMFVGVGPARVRDLFALARKNAPCILFIDE VNGSEFLEMFVGVGPARVR LFVLARKNAPCILFIDE VNGSEFLEMFVGVGPARVRPMFAMARKNAPCILFIDE VSGSEFLEMFVGVGPSRVRPMFAMARKHAPCILFIDE VSGSEFLEMFVGVGPARVRPMFSMARKNSPCILFIDE VSGSEFVEMFVGVGASRVRDLFTQARSMAPSIIFIDE
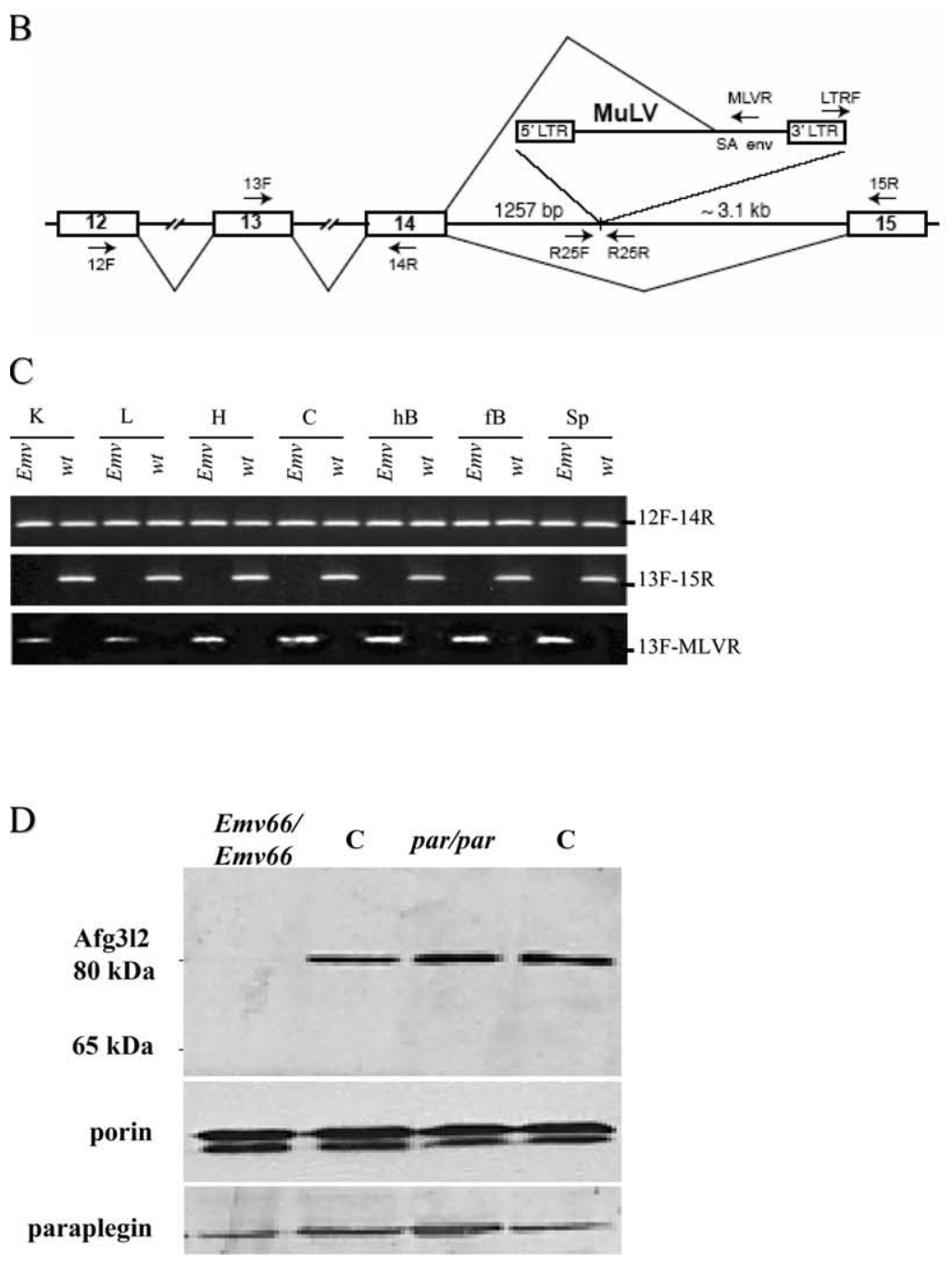

Figure 1. Molecular characterization of $A f g 3 / 2$ mutant models. A, Partial sequence alignment of AAA domain of $A f g 3 / 2$ orthologs. The mutant arginine residue (R389G, boxed) of $A f g 3 / 2^{\text {par/par }}$ mouse is highly conserved among different species. $\boldsymbol{B}$, $S$ chematic representation of provirus integration into intron 14 of the $A f g 3 / 2$ gene. Arrows identify primers used (for details, see Materials and Methods). C, RT-PCR showing Afg3/2 ${ }^{\text {Emv66/Emv66 }}$ and wild-type alleles expression in different tissues. K, Kidney; $L$, liver; $H$, heart; $C$, cerebellum; $h B$, hindbrain; $f B$, forebrain; Sp, spinal cord. $D$, Western blot analysis on isolated mitochondria from Afg3/2 $2^{\text {Emv66/Emv66 }}\left(\right.$ Emv66/Emv66), Afg3/2 ${ }^{\text {par/par }}$ ( par/par), and syngenic control mice (C). Anti-porin antibody is used to normal-

itz, 1985) on fresh-frozen muscle of mutant mice and control at postnatal day $14(\mathrm{P} 14 ; n=4)$.

Antibodies. Anti-Hsp60 mAb was from Nventa Biopharmaceuticals (San Diego, CA); anti-OxPhos complex I 39 kDa subunit, anti-complex III (25.6 kDa subunit), and anti-porin $\mathrm{mAb}$ were from Invitrogen (Eugene, OR); and anti-complex I (20 kDa subunit) was from Acris Antibodies (Hiddenhausen, Germany).

Mitochondria isolation. Mitochondria were isolated by differential 


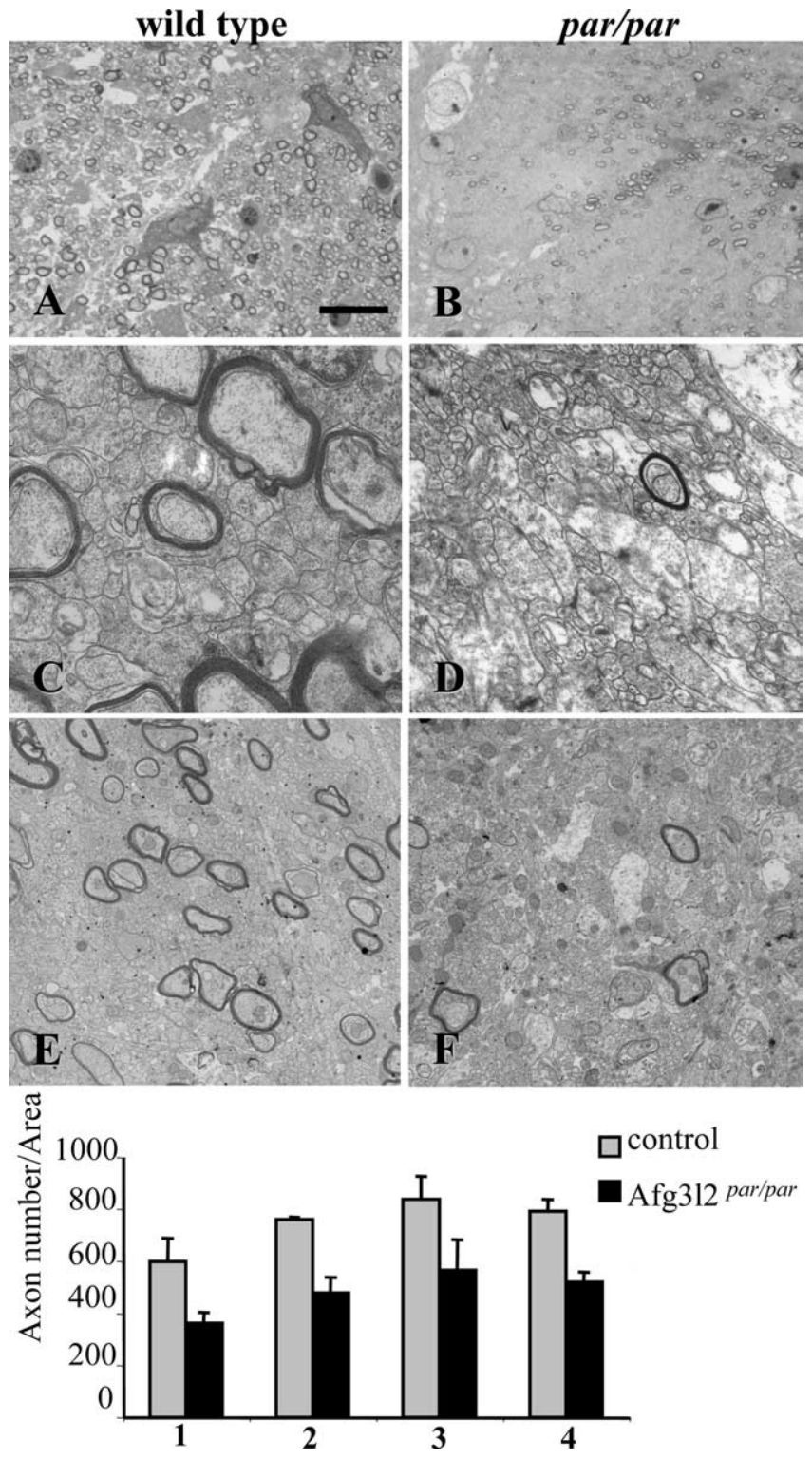

Figure 2. Reduction of myelinated fibers in corticospinal tract. $A, B$, Semithin sections of spinal cord of Afg $312^{\text {par } / \text { par }}$ mice and controls at P14. In the mutants the number of myelinated axons is dramatically reduced in the corticospinal tract. $C, D$, Ultrathin sections of corticospinal tract of Afg $3 / 2^{\text {par/par }}$ mice and controls at P7. Myelinated axons are dramatically less represented in the mutants. $E, F, A t$ P12, ultrastructural analysis confirms the impressive reduction of myelinated fibers in the mutants compared with controls, where almost all axons are myelinated. Scale bar (in $\boldsymbol{A}$ ): $\boldsymbol{A}, \boldsymbol{B}, 20 \mu \mathrm{m} ; \boldsymbol{C}, \boldsymbol{D}, 1 \mu \mathrm{m} ; \boldsymbol{E}, \boldsymbol{F}, 2 \mu \mathrm{m}$. The graph shows counts of total myelinated axons in spinal cord from Afg3/2 $2^{\text {parpar }}$ mice and controls at P14. A significant loss of axons in the mutant is visible. Comparing statistics are given as spinal region/ $t$ test $p$ value: 1 , corticospinal tract $/ 0.014 ; 2$, anterior funiculi $/ 0.016 ; 3$, anterolateral funiculi/ $/ 0.008 ; 4$, fasciculus gracilis/0.014. (supplemental text, available at www.jneurosci.org as supplemental material). Error bars represent $\pm \mathrm{SD} ; n=3$.

centrifugation from different tissue homogenates (Robinson, 1996). In brief, tissues were homogenized in an appropriate isotonic buffer $[0.25 \mathrm{M}$ sucrose, $20 \mathrm{~mm}$ 3-( $N$-morpholino)propanesulfonic acid (MOPS), $\mathrm{pH}$ 7.2, 1 mм EDTA, 0.1\% BSA fatty acid free and digitonin $0.1 \mathrm{mg} / \mathrm{ml}$ ] using a glass-Teflon homogenizer. Cell debris and nuclei were pelleted twice by centrifugation at $2500 \mathrm{~g}$ for $5 \mathrm{~min}$ at $4^{\circ} \mathrm{C}$. Supernatants were centrifuged at $12000 \mathrm{~g}$ for $25 \mathrm{~min}$ at $4^{\circ} \mathrm{C}$ and the mitochondrial pellet was resuspended in an isotonic buffer ( $0.5 \mathrm{~m}$ sucrose, $20 \mathrm{~mm}$ MOPS, pH 7.2, $1 \mathrm{~mm}$ EDTA).

Respiratory chain activity. Tests for respiratory chain defects were essentially performed as described by Robinson (Robinson, 1996). In brief, isolated mitochondria were incubated at $37^{\circ} \mathrm{C}$ for $30 \mathrm{~min}$ in a respiratory buffer $(0.25 \mathrm{~m}$ sucrose, $20 \mathrm{~mm}$ MOPS, $1 \mathrm{~mm}$ EDTA, $5 \mathrm{~mm}$ inorganic phosphate, $0.1 \%$ BSA fatty acid free, and $1 \mathrm{~mm}$ ADP, pH 7.4) containing specific substrates and inhibitors of the respiratory chain complexes. By providing pyruvate/malate (5 and $1 \mathrm{~mm}$, respectively) and glutamate/ malate ( 5 and $1 \mathrm{~mm}$, respectively), we stimulated ATP synthesis dependent on complexes I, II, III, IV, and V. Using a complex I inhibitor, $1 \mathrm{~mm}$ rotenone, and $10 \mathrm{~mm}$ succinate, we measured ATP production that is dependent on complexes II, III, IV, and V. Finally, incubating mitochondria with $2 \mathrm{~mm}$ antimycin A, an inhibitor of complex III, and tetramethyl-p-phenylenediamine (TMPD)/ascorbate $(0.1$ and $2 \mathrm{mM}$, respectively), we evaluated the ATP level resulting from the activity of complexes IV and V. ATP production was measured by luminometric assay.

Luminometric assay of ATP. ATP concentration was determined with the luciferin-luciferase method as described previously (Ronner et al., 1999); in brief, the assay solution was prepared as follows: $250 \mathrm{~mm}$ glycylglycine, 2 mм EGTA, $2 \mathrm{~mm} \mathrm{MgCl}_{2}, 0.4 \mathrm{~g} / \mathrm{L}$ BSA fatty acid free, $7.5 \mathrm{~mm}$ DTT, $15 \mu \mathrm{M}$ luciferin, and $10 \mu \mathrm{g} / \mathrm{ml}$ luciferase. Isolated mitochondria were lysed with the ATP lysis buffer $(0.2 \mathrm{M} \mathrm{NaOH}$ and $0.5 \mathrm{~mm}$ EDTA), and an aliquot of the obtained extract was diluted with the ATP dilution buffer $(0.1 \mathrm{M} \mathrm{NaOH}$ and $0.5 \mathrm{~mm}$ EDTA). Twenty microliters of this mixture were added to $100 \mu \mathrm{l}$ of the assay solution, and the ATP content was measured by luminometer. Results are expressed as nanomoles of ATP per milligram of protein. Data, in triplicates, are indicated as mean of four independent experiments \pm SD. Four mice per genotype were analyzed. Two-tailed $t$ test was applied for significance calculation.

COX activity assay. COX activity assay was performed using a commercial kit (CYTOCOX1; Sigma, St. Louis, MO) and following manufacturer instructions.

$B N-P A G E$ and complex I in situ activity staining. Isolated mitochondria were solubilized by dodecyl maltoside ( $2 \%$ final concentration) and centrifuged at $12000 \times g$ for $25 \mathrm{~min}$ at $4^{\circ} \mathrm{C}$. Supernatants were loaded on a linear 5-13\% gradient polyacrylamide gel. Complex I in situ activity staining was performed by incubation of the gel in $0.1 \mathrm{M}$ Tris- $\mathrm{HCl}, 0.14$ $\mathrm{mm} \mathrm{NADH}$, and $1 \mathrm{mg} / \mathrm{ml}$ nitroblue tetrazolium, $\mathrm{pH} 7.4$; the reaction was performed at room temperature and stopped after 30 min by $45 \%$ methanol and 10\% acetic acid (Jung et al., 2000). Gels were transblotted onto a nitrocellulose membrane and immunodecorated with anti-39 $\mathrm{kDa}$ and anti-core2 (25.6 kDa subunit) antibodies (Schagger et al., 1994). Band quantifications relative to in situ activity staining gels and Western blots were performed by densitometric analysis. Four mice per genotype were analyzed. Two-tailed $t$ test was used for significant calculation.

\section{Results}

\section{Murine models defective in Afg3l2}

We describe two different $A f g 3 l 2$ genetic models, both showing severe motor impairment that resembles human motoneuron diseases.

The first mutation, paralysé (symbol par; Mouse Genome Informatics Accession number, 97480), occurred at the Institute Pasteur in C57BL/6 strain (Duchen et al., 1983; Blondet et al., 1989). We localized par mutation on mouse chromosome 18 between markers D18Mit140 and D18Mit141 and further narrowed to $630 \mathrm{~kb}$ by haplotype analysis of two inter-subspecific F2 offspring (see supplemental Materials and Methods, available at www.jneurosci.org as supplemental material). This critical interval harbors a total of 11 genes, 10 of which were considered candidates based on their expression patterns (Genomics Institute of the Novartis Research Foundation-Symatlas, http://symatlas.gnf.org). cDNA libraries were prepared from par/par and $+/+$ littermates and sequencing was systematically performed for all exons of the positional candidate genes. This led us to detect a missense mutation (a $\mathrm{C}$ to $\mathrm{G}$ transversion) in exon 10 of the $A f g 3 l 2$ gene leading to the substitution of an arginine by a glycine residue $(\mathrm{R} 389 \mathrm{G})$ in the highly conserved AAA domain of the molecule (Fig. 1A). 
A second Afg3l2 model was discovered after a novel ecotropic murine leukemia proviral reinsertion in the $\mathrm{MEV} / 2 \mathrm{TyJ}$ strain (Taylor and Rowe, 1989; Taylor and Frankel, 1993) and was assigned the allele symbol Emv66 for ecotropic murine leukemia virus insertion 66. As shown in Figure $1 B$, the Emv66 provirus integrated within intron 14 of the $A f g 3 l 2$ gene. The genomic sequences flanking the provirus were intact with the exception of a fournucleotide (AATT) duplication at the site of insertion. To determine the effect of the murine leukemia virus (MuLV) insertion on $A f g 3 l 2$ gene expression, we amplified cDNA from several tissues using primers upstream of the insertion (exons 12-14) and saw no difference in expression between homozygous Emv66 and wild-type littermates in any examined tissue (Fig. $1 C$, top, 12F_14R). In contrast, reverse transcription (RT)-PCR products corresponding to the mature mRNA from exons 13-15 (Fig. 1C, middle, 13F_15R) across the MuLV insertion were undetectable in Emv66 homozygotes. When a reverse primer within the MuLV envelope gene (MLVR) was paired with an $A f g 3 l 2$ exon 13 forward primer, a mutant-specific spliced product could be detected by RT-PCR in all tissues (Fig. 1C, bottom, 13F_MLVR). The resulting fusion transcript is predicted to truncate the AFG3L2 protein at residue 592, thus deleting the final 210 amino acids and adding 24 novel amino acids before terminating at a TAG stop codon.

To facilitate these models' nomenclature, we indicate hereafter the homozygous missense mutant as $A f g 3 l 2^{\text {par/par }}$ and the homozygous null mutant as Afg $312^{\text {Emv66/Emv66 }}$.

Western blot analysis performed on tissue mitochondrial preparations using an AFG3L2 antibody (Atorino et al., 2003) confirms that both the full-length and the truncated AFG3L2 protein (80 and $65 \mathrm{kDa}$, respectively) are absent in $A f g 3 l 2^{\text {Emv66/Emv66 }}$ mice. On the contrary, in Afg3l2 $2^{\text {par/par }}$ mice the AFG3L2 protein is detected normally, with no difference in expression level, compared with controls. Western blot analysis performed using an antibody against paraplegin reveals that the absence of AFG3L2 does not affect paraplegin expression in mitochondria enriched from brain (Fig. $1 D$ ) and liver (data not shown).

\section{Early onset paraparesis and tetraparesis in Afg 312 mutant mice}

The phenotype of the two mutant mouse models is strikingly similar and extremely severe. Heterozygous $A f g 3 l 2^{+/ E m v 66}$ and Afg $3 l 2^{+/ p a r}$ mice have a normal appearance and fertility. Homozygous $A f g 3 l 2^{\text {Emv66/Emv66 }}$ and $A f g 3 l 2^{\text {par/par }}$ are significantly smaller than their littermates at 1 week of age and display a rapidly progressive loss of motor function in all limbs by 12-14 d. As the disease progresses, they lose the ability to support their own weight or turn themselves over when placed on their back and exhibit a typical posture with over extension of all limbs and uncoordinated movements (supplemental Movies 1, 2, available at www.jneurosci.org as supplemental material). They rarely survive beyond $16 \mathrm{~d}$ of age, when they are completely paralyzed. The mutant phenotype is genetically recessive and fully penetrant.
The reciprocal crossing of a male $A f g 3 l 2^{+/ E m v 66}$ and a female $A f g 3 l 2^{+/ p a r}$, or vice versa, gives identical affected compound heterozygous littermates.

\section{Widespread reduction of myelinated fibers in spinal cord}

Considering the rapid progression and the severity of the disease affecting $A f g 3 l 2$ mutant mice, we have performed an extensive morphological analysis of CNS and PNS on both strains. LM and EM examination of $A f g 3 l 2^{\text {Emv66/Emv66 }}$ and $A f g 3 l 2^{\text {par/par }}$ mutant mice reveals identical morphological alterations in axons and cell bodies of CNS and PNS motor and sensory neurons.

Spinal cords from both mutant mice show reduced diameters compared with control littermates at P14 (data not shown). As shown in Figure 2, semithin sections of spinal cord from Afg $312^{\text {par/par }}$ mice show a marked and widespread reduction of myelinated fiber density. The remaining myelinated fibers barely reach the $2 \mu \mathrm{m}$ diameter, hence, supporting a specific loss of large axons. At lumbar level, the observed abnormalities are most prominent in the dorsal columns, predominantly, but not exclusively, in the corticospinal tract (Fig. 2 B). This reduction of myelinated fibers is also detected in the external regions of the anterior and anterolateral funiculi (supplemental Fig. $1 B, D$, available at www.jneurosci.org as supplemental material). In rodents these extrapyramidal motor axons are responsible for the major motor control, as the murine corticospinal tract is mainly polysynaptic and composed of shorter axons (Nieuwenhuys et al., 1998). Similar neuropathological signs are also visible in the medial part of the dorsal funiculi, where sensory neuron axons ascend within the fasciculus gracilis (supplemental Fig. $1 F$, available at www. jneurosci.org as supplemental material). Quantitative analysis confirms the marked reduction of myelinated fiber density in the analyzed spinal areas (Fig. 2, graph).

We observe no evidence of axonal degeneration, such as swollen or collapsed axons. Moreover, the evaluation of a possible glial outgrowth secondary to axonal degeneration resulted in no 

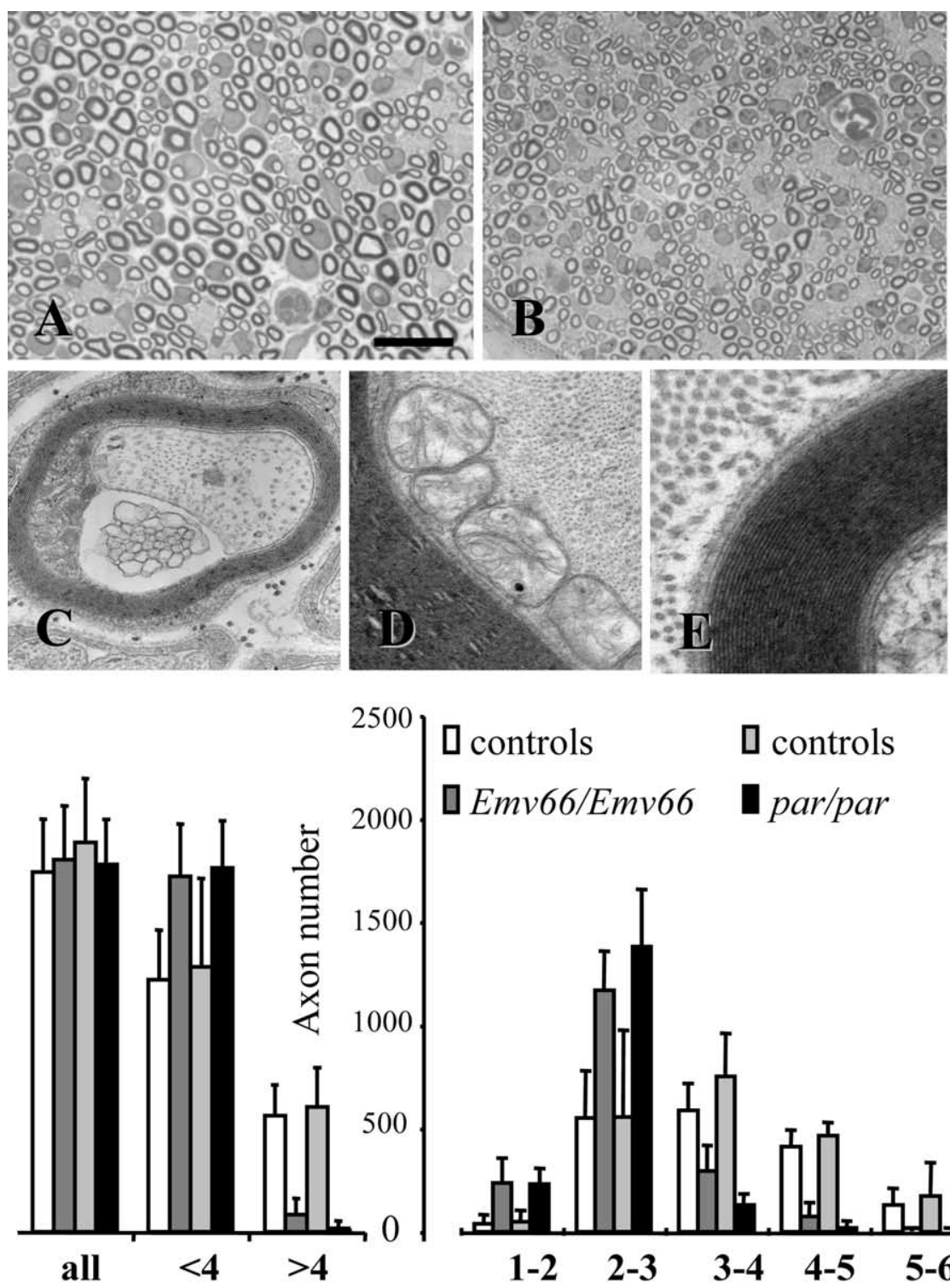

\section{Axon diameter}

$(\mu \mathrm{m})$

Figure 4. Sciatic nerve lacks large caliber axons. $A, B$, Semithin sections of sciatic nerve of control $(\boldsymbol{A})$ and $A f g 3 / 2^{\text {par par }}$ mouse $(\boldsymbol{B})$ at P14. The reduced axonal diameter is evident. $\boldsymbol{C}, \boldsymbol{D}$, EM shows Schwann cell infoldings into the axon $(\boldsymbol{C})$, and clustering of swollen mitochondria with poor residual cristae $(\boldsymbol{D})$ is detected. $\boldsymbol{E}$, At this age, compact myelin sheaths appear normal, with correct periodicity of major dense and intraperiod lines. Left graph shows counts of total, small ( $<4 \mu \mathrm{m}$ diameter), and large ( $>$ $4 \mu \mathrm{m}$ diameter) myelinated axons in sciatic nerve from Afg3/2 ${ }^{\text {par/par }}$, Afg $312^{\text {Emv66/Emv66 }}$, and control mice. A significant loss of large caliber axons appears in both mutants. Right graph shows averaged distribution of axon diameters from sciatic nerve of Afg $3 / 2^{\text {par/par }}$ and Afg3/2 ${ }^{\text {Emv66/Emv6 }}$ mice and controls. Axonal diameter distribution is skewed to 2-3 $\mu \mathrm{m}$ in the mutants. Statistical comparison with controls are as follows (class of axon diameter/t test $p$ value): Afg3/2 $2^{\text {Emv66/Emv66 }},<4 \mu \mathrm{m} / 0.005,>4$ $\mu \mathrm{m} / 0.021 ; A f g 312^{\text {par } / \text { par }},<4 \mu \mathrm{m} / 0.004,>4 \mu \mathrm{m} / 0.005$. Error bars represent $\pm S D ; n=3$. Scale bar (in $A$ ): $A, B, 20 \mu \mathrm{m} ; C, 0.5$ $\mu \mathrm{m} ; \boldsymbol{D}, \boldsymbol{E}, 0.2 \mu \mathrm{m}$.

differences between mutants and controls (GFAP immunofluorescence) (data not shown). The loss of myelinated fibers observed in $A f g 3 l 2$ mutant mice is thus attributable to dysfunctional axonal development rather than to axonal degeneration. In fact, ultrathin sections of corticospinal tract at P1 show similar number of unmyelinated axonal processes in mutant and control mice (fiber count per area, $113 \pm 8 \mathrm{SD}$ in $A f g 3 l 2^{\text {Emv66/Emv66 }} ; 109 \pm 7 \mathrm{SD}$ in controls), whereas at P7 only few myelinated axons are present in the mutants (Fig. 2D)

This delay in myelination is confirmed at P14 at EM magnification, when most of the axons are myelinated in the controls, differently from the mutants that show $\sim 60 \%$ of myelinated fibers and numerous small unmyelinated axons, likely too small to trigger the myelin ensheathing process (Fig. $2 F$ ). Identical results were obtained for $A f g 3 l 2^{E m v 66 / E m v 66}$ mice (data not shown).

\section{Massive vacuolization of motoneuron cell bodies, dorsal root ganglia and Purkinje cells}

The number of neurons in spinal cord anterior horn, sensory dorsal root ganglia (DRGs) and the number of Purkinje cells is conserved in both $A f g 3 l 2^{\text {Emv6r/Emv66 }}$ and Afg $312^{\text {par/par }}$ mutants compared with controls at P14 (neuron count per area of mutants vs control \pm SD in anterior horn, DRGs, and Purkinje cells are, respectively, $7.75 \pm 1.29$ vs $7.4 \pm 1 ; 20.2 \pm 1.86$ vs $20 \pm$ $2.58 ; 9 \pm 1.67$ vs $9 \pm 1.41)$. In fact, we find no evidence of neuronal loss, suggesting that this is not a primary event in these models. Multiple large vacuoles with clear content cluster in the cytoplasm (Fig. $3 B, F, J)$. As shown by EM examination, the observed vacuoles are suggestive of mitochondrial origin (Fig. 3D,H,L). Displacement of the inner membrane, cristae disruption and increased volume of the intermembrane space are observed in swollen mitochondria.

\section{Peripheral nerves lack large caliber axons}

Although AFG3L2 is a structural component of the $m$-AAA protease complex that is aberrant in HSP, a pure primary motoneuron disease, the severity of the $A f g 3 l 2$ mutants' phenotype has prompted us to investigate the peripheral nervous system as well. Again, in both $A f g 3 l 2^{\text {Emv66/Emv66 }}$ and $A f g 3 l 2^{\text {par/par }}$ mice large caliber axons are dramatically less represented in the mutant sciatic nerve (Fig. $4 B$, relative to Afg3l2 $2^{\text {par/par }}$ mice) (data not shown for

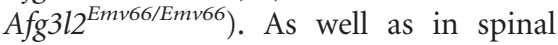
cord, the total number of axons between both mutant strains and respective controls is conserved at P14, thus indicating no axonal loss in the sciatic nerve (Fig. 4, left graph). However, the relative proportion of small $(<4 \mu \mathrm{m}$ diameter $)$ and large $(>4$ $\mu \mathrm{m}$ diameter) axons changes significantly with a clear shift to smaller calibers, demonstrating that the growth of large diameter axons is severely affected in the mutants (Fig. 4, right graph). EM on $A f g 3 l 2^{\text {par/par }}$ mice shows no signs of active axonal degeneration compared with controls. However, as shown in Figure $4 C$, signs 


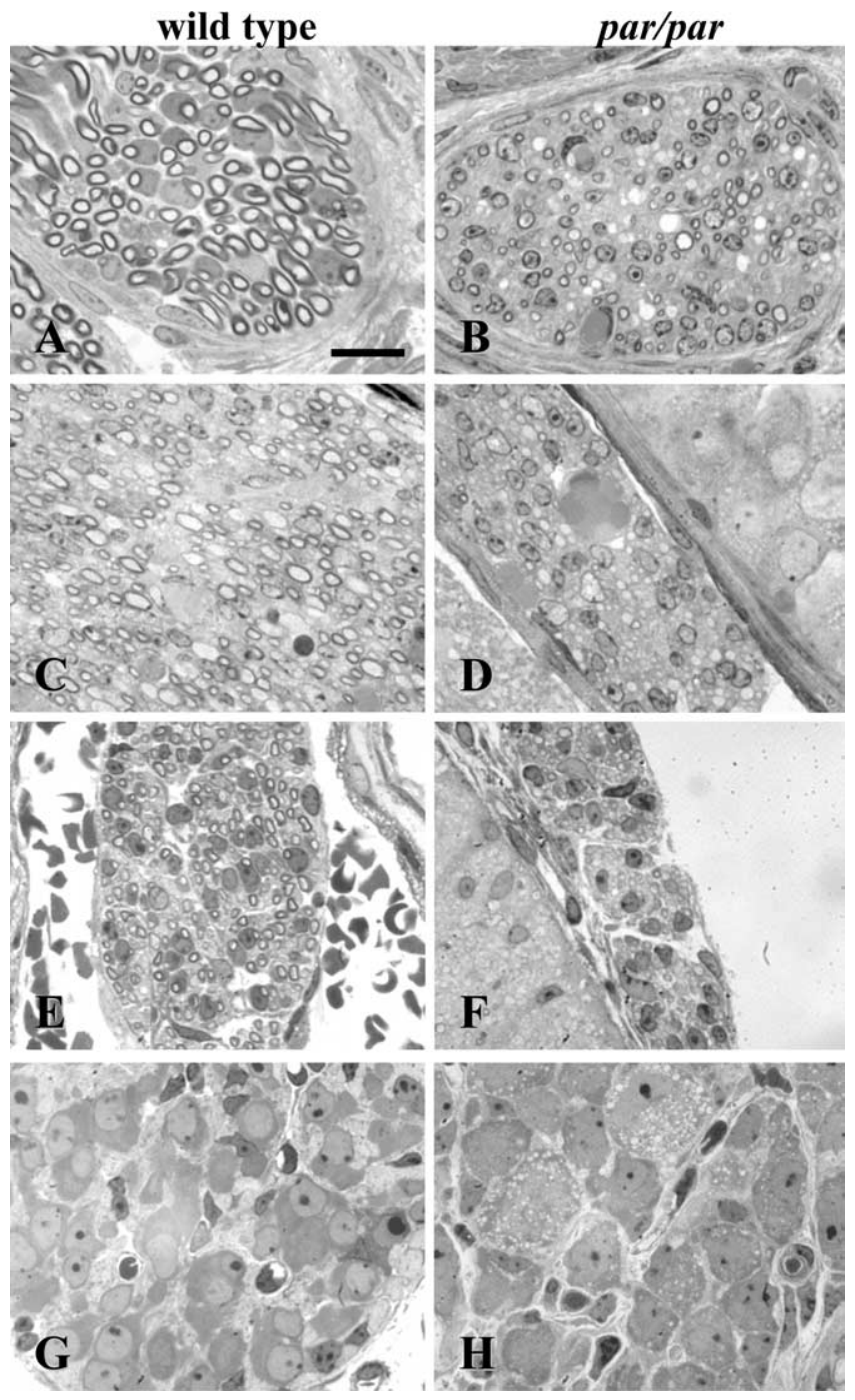

Figure 5. Delayed myelination is preceded by neuronal cell body vacuolization. $\boldsymbol{A}-\boldsymbol{H}$, Semithin sections of sciatic nerve at $\mathrm{P5}(\boldsymbol{A}, \boldsymbol{B})$, dorsal roots at $\mathrm{P5}(\boldsymbol{C}, \boldsymbol{D})$, ventral roots $(\boldsymbol{E}, \boldsymbol{F})$ at $\mathrm{P} 1$, and DRGs at P1 $(\boldsymbol{G}, \boldsymbol{H})$ of controls (left) and Afg3/2 2 ar/par (right). Reduced number of myelinated fibers is evident in sciatic nerve $(\boldsymbol{B})$ and dorsal and ventral roots ( $\boldsymbol{D}$ and $\boldsymbol{F}$, respectively). In the mutants, mitochondrial vacuolization is already present in DRGs at P1 $(\boldsymbol{H})$. Scale bar, $20 \mu \mathrm{m}$.

of axonal damage such as Schwann cell infoldings into the axoplasm and disintegration of adaxonal myelin lamellae are detected. Altered mitochondria are often closely associated in clusters or aligned in rows and display damaged cristae and swelling (Fig. 4D). Compact myelin sheaths appear normal with correct periodicity of major dense and intraperiod lines in all fibers at P14 (Fig. 4E). However, as occurring in spinal cord, the myelination process is delayed in sciatic nerve at $\mathrm{P} 5$ in the mutants, which show a reduced number of myelinated fibers compared with controls ( $31.2 \pm 4.6 \mathrm{SD}$ vs $76.7 \pm 2.1$ per area) (Fig. $5 B)$. This is even more pronounced in the dorsal roots at P5 (Fig. 5D) and in ventral roots at P1 (Fig. $5 F$ ), thus confirming a defect in axonal development also in the PNS. Remarkably, the cytoplasmic vacuolization is already present in DRGs at P1 when $76 \%$ of cells are already vacuolated (Fig. $5 \mathrm{H}$ ). Identical findings were observed in Afg3l2 ${ }^{\text {Emv66/Emv66 }}$ mice as well (data not shown).

\section{Decrease of neurofilament content}

Our results demonstrate that axonal radial growth is affected by Afg3l2 mutations. Because NFs are major determinants of axonal diameter (de Waegh et al., 1992), we hypothesize that NF content or structure may be altered in our models. Quantitative evaluation of NF density reveals a significant decrease in both mutant mice $\left(126.1 \pm 47.4 \mathrm{NFs} / \mu \mathrm{m}^{2}\right.$ in $A f g 3 l 2^{\text {par/par }}$ and $137.3 \pm 33.9$ in Afg3l2 ${ }^{\text {Emv66/Emv66 }}$ axons vs $206.6 \pm 58.4$ in control axons) (Fig. 6, graph). Furthermore, in contrast with the ordered and regularly spaced NF architecture in unaffected axons, NFs appear densely packed in clusters and unevenly distributed in mutant axons, leaving empty areas within the axoplasm (Fig. $6 B, C$ ). Instead, microtubules density is invariant between mutants and controls (data not shown).

\section{Non-neuronal tissues are not primarily altered in Afg3l2 mutants}

Morphological evaluation of muscle specimens from both mutant mice at P14 (Fig. 7 relative to $A f g 3 l 2^{\text {par/par }}$ mice) (data not shown for $A f g 32^{\text {Emv66/Emv66 }}$ ) shows a general decrease of fiber size but no signs of inflammation and/or degeneration/necrosis. Perimysial and endomysial connective tissue are not increased. Muscle fibers with centrally placed nuclei are sporadically seen (Fig. 7C). A percentage of fibers show accumulation of mitochondria, a red-purple appearance with the modified trichrome stain (Fig. $7 B), \operatorname{SDH}(E)$, and $\operatorname{COX}(F)$, by histochemical analysis. In these fibers, the enlarged mitochondria occupy the entire central part of the muscle fiber as confirmed by ultrastructural analysis displaying mitochondria distorted cristae, electron dense bodies and, in some cases, lipid accumulation (Figs. 7G,H). The quantitative evaluation of these findings in different muscles shows that aberrant fibers with centrally positioned nuclei or mitochondria are quite rare (Fig. 7, graph). Ultrathin sections of liver show no differences in mitochondrial morphology in controls and Afg3l2 $2^{\text {par } / p a r}$ mice (Fig. $7 I, J$, respectively). The same results were obtained for $A f g 3 l 2^{E m v 66 / E m v 66}$ (data not shown). Together, these data indicate that $A f g 3 l 2$ mutation is primarily affecting neuronal tissues and the evident decrease of muscle fiber size is of neuronal origin.

\section{Impaired respiratory complex I and III activity in Afg3l2 mutant mice}

We have demonstrated previously that HSP fibroblasts lacking the $m$-AAA complex have a reduced respiratory complex I activity (Atorino et al., 2003). To investigate the role of AFG3L2 in mitochondrial metabolism, we tested ATP production in brain of both $A f g 3 l 2^{\text {par/par }}$ and $A f g 3 l 2^{\text {Emv66/Emv66 }}$ mutant mice and controls in the presence of different substrates and inhibitors. The basal activity of the respiratory chain is similar in controls and mutants. We detect a substantial reduction of ATP synthesis in mutant mitochondria using pyruvate and glutamate, which stimulate the activity of the overall respiratory chain complexes, and also using succinate, which stimulates the activity of complexes II, III, IV, and V (Fig. 8A). The ATP level measured in presence of ascorbate is the same in mutants and in controls, suggesting a comparable activity of complexes IV and V (nmol of ATP/mg of mitochondrial proteins $\pm \mathrm{SD}, A f g 3 l 2^{\text {par } / p a r}$ vs controls, $5.0 \pm 1.6$ vs $4.7 \pm 0.9 ; A f g 312^{\text {Emv66/Emv66 }}$ vs controls, $6.2 \pm 0.6$ vs $6.15 \pm 0.3$ ) (Fig. $8 \mathrm{~A}$ ). We excluded defects in complex IV (COX) activity, also assaying the oxidation of cytochrome $c$ with a specific spectrophotometric assay (data not shown). These results correlate with a possible impairment of complex I, II or III activity. Therefore we used blue native (BN)-PAGE to evaluate the enzymatic activity of complex I and BN-PAGE followed by immunoblot to evaluate the correct assembly of the respiratory chain complexes. By combining BN-PAGE with an in situ activity staining reaction, 

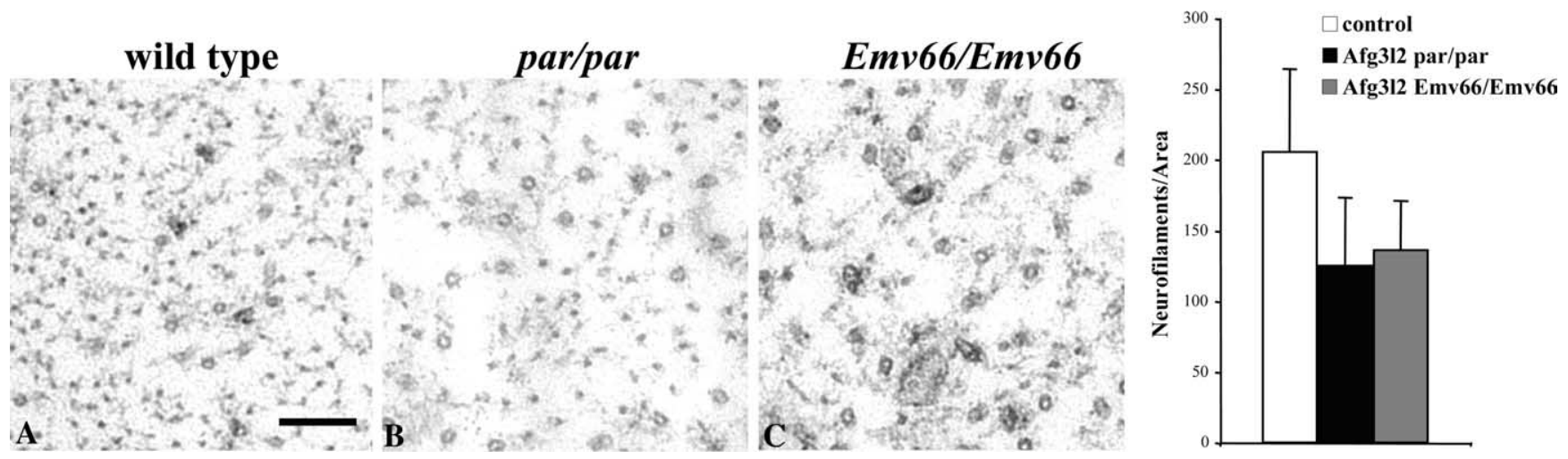

Figure 6. $\boldsymbol{A}-\boldsymbol{C}$, Alteration of axoplasm structure. Sciatic nerve ultrathin preparations of control $(\boldsymbol{A}), A f g 3 / 2^{\text {par/par }}$ mouse $(\boldsymbol{B})$, and $A f g 3 / 2^{\text {Emv66/Emv66 }}(\boldsymbol{C})$ at P14. In the mutants, axoplasm appears poorly organized compared with the more even distribution observed in controls. Scale bar, $100 \mathrm{~nm}$. Quantitative analysis (graph) reveals a reduction of neurofilament density in both mutants compared with controls. Afg3/2 ${ }^{\text {par/par }}, p=0.00001 ; A f g 3 / 2^{\text {Emv66/Emv66 }}$ versus controls, $p=0.002$ ( $t$ test). Error bars represent $\pm S D$. An average of six axons in true cross sections were randomly selected for each genotype $(n=2)$.

we observe a statistically significant decrease in complex I activity in brain-derived mitochondria from both mutant mice compared with controls (Fig. $8 \mathrm{~B}$ ). BN-PAGE followed by immunoblot reveals that the reduced complex I activity is attributable to a decreased amount of assembled complex I (Fig. 8C), despite the unchanged intramitochondrial availability of unassembled complex I building blocks (we tested the $39 \mathrm{kDa}$ protein) (supplemental Fig. $2 B$, available at www.jneurosci.org as supplemental material). According to ATP synthesis data, we also identified a decreased amount of complex III (Fig. 8D). The same results were obtained for mitochondria derived from spinal cord (data not shown).

\section{Mitochondrial protein synthesis is not impaired in mutant mice}

Previous studies identified MrpL32, a subunit of mitochondrial ribosomes, as a proteolytic substrate of the $m$-AAA protease and assigned it a crucial function controlling mitochondrial protein synthesis in both yeast and mammals. In particular, it has been shown that mitochondrial translation is significantly impaired in paraplegin-deficient liver mitochondria and reduced by $50 \%$ when compared with wild type (Nolden et al., 2005). Based on these data, we investigated whether the reduced amount of complex I and complex III observed in Afg 312 mutant mice could reflect either a decreased availability of respiratory complexes' building blocks or an insufficient assembling. We thus analyzed the efficiency of protein synthesis in isolated $A f g 3 l 2$-deficient mitochondria. Both $A f g 3 l 2$ mutant strains show no impairment of mitochondrial protein synthesis in different tissues, e.g., brain (supplemental Fig. $2 A$, available at www.jneurosci.org as supple-

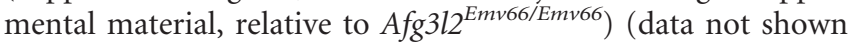
for $A f g 3 l 2^{\text {par/par }}$ ) and liver (data not shown). Western blot analysis using antibodies against complex I subunit of mitochondrial (20 kDa subunit) and nuclear (39 kDa subunit) origins confirms normal mitochondrial translation and import efficiency in both Afg3l2 mutants (supplemental Fig. $2 B$, available at www.jneurosci.org as supplemental material).

\section{Discussion}

\section{Mutant phenotype}

The remarkably overlapping and severe phenotype of the two Afg3l2 genetic mutants, which differ in both genetic background and causal mutations, denotes that AFG3L2 is a mitochondrial metalloprotease with a key function in neurons. Our observa- tions derive from the phenotypic and biochemical analysis of two different $A f g 3 l 2$ genetic models. We identified the genetic cause (i.e., a single amino acid-R389G-mutation occurring in the core AAA domain of AFG3L2) that arose in the C57 background leading to the spontaneous par/par mutant. In addition, we developed a null AFG3L2 mutant $\left(A f g 3 l 2^{\text {Emv6/Emv66 }}\right)$ by insertional mutagenesis on FVB murine background. As expected, we confirmed the presence and stability of the missense AFG3L2 protein in the Afg $3 l 2^{\text {par/par }}$ strain and the complete absence of the protein in the null $A f g 3 l 2^{\text {Emv66/Emv66 }}$ mutant.

Similar to the paraplegin-deficient mouse model, Afg $3 l 2 \mathrm{mu}$ tants neuropathology establishes axonopathy as a recurrent theme for $m$-AAA alterations. However, Afg $3 l 2$ mice present a failure in axonal development that results in early lethality, whereas the paraplegin-null mouse shows late onset axon degeneration.

This is first evident by the dramatic decrease of myelinated fiber density in the spinal cord, where the total number of axons is conserved but only few and small myelinated axons are present. These alterations have been found both in motor and in sensory areas at P12 and are even more pronounced at P7, hence, indicating an impairment of axonal development. We could exclude that these defects are attributable to neuronal loss and axonal degeneration, because the number of neuronal cell bodies is conserved, and typical morphological markers, such as swollen/degenerating axons and reactive glia, are absent in mutant mice.

In accordance with the spinal cord data, in the sciatic nerve at P12 the total number of fibers is conserved but large caliber axons are missing, with a shift of the distribution of diameter frequency toward classes of thinner axons. At P5 we observe a decreased number of myelinated axons and an increased fraction of uncommitted nonmyelinating or premyelinating Schwann cells compared with controls, suggesting a delay in the myelination process in the PNS. This hypothesis is also supported by the marked reduction of myelinated fibers in ventral and in dorsal roots at P5 and at P1. Ultrastructural analysis of sciatic nerve also reveals many signs of axonal damage, such as disorganized cytoskeletal structure associated to altered neurofilament density and distribution, the presence of aberrant organelles and Schwann cells invagination within the axoplasm.

Collectively, these findings strongly suggest that nonfunctional AFG3L2 causes an axonal development failure in CNS and 
PNS. Processes unique to either oligodendrocytes or Schwann cells may account for the different timing of axon myelination observed in CNS and PNS (Trapp et al., 2004).

\section{Mitochondrial effect of AFG3L2 mutations}

The lack of functional AFG3L2 has deep effects on mitochondrial morphology and metabolism. Most mitochondria are morphologically altered in CNS and PNS of both mutants, where swollen giant mitochondria with damaged cristae can be observed. These aberrant organelles are generally clustered in proximity of the nucleus or the plasma membrane. This phenomenon, together with the presence of several large vacuoles of mitochondrial origin, is primarily observed in neuronal cell bodies of spinal cord anterior horns, in dorsal root ganglia and in Purkinje cells, the latter indicating a cerebellar involvement.

Morphological analysis of nonneuronal tissues, i.e., liver and skeletal muscle, demonstrates that the nervous system is uniquely affected by $A f g 3 l 2 \mathrm{mu}-$ tations. Indeed, no altered mitochondria are present in liver and only rare muscle fibers display abnormal accumulation of mitochondria, likely because of the primary axonal defect.

Mitochondrial morphological alterations correlate with impaired mitochondrial metabolism in the absence of functional AFG3L2. In fact, we detect a dramatic reduction of respiratory complex I and III activities, which correspond to stoichiometric limitation of the enzymatic complexes. Insufficient assembling, rather than decreased availability of building blocks of respiratory complexes, plays a central role in this deficit of respiratory activities, because neither synthesis of mitochondria-encoded subunits nor import of nuclear-encoded proteins is impaired. Therefore, our data suggest a role for AFG3L2 in effectively assembling com-

plexes I and III. Remarkably, a defect in the assembly of respiratory complex I was associated to organellar dysfunction in HSP patients (Atorino et al., 2003).

These results partially diverge from previous findings on paraplegin-deficient mice showing a slightly decrease of ATP synthesis in the spinal cord of 23 months old Spg $7^{-1-}$ mice (Ferreirinha et al., 2004) and an impairment of mitochondrial translation in the liver (Nolden et al., 2005). The phenotypic differences between $A f g 3 l 2$ and paraplegin mutants can be explained at the molecular level by the different ability of the two $m$-AAA proteins in organizing supramolecular complexes. In fact, AFG3L2 can homo-oligomerize both in human and in mouse, whereas paraplegin is uniquely found as part of hetero-oligomeric complexes. Homo-oligomeric AFG3L2 m-AAA complexes are present in physiological conditions and can exert proteolytic ac- tivity (Koppen et al., 2007). These data indicate AFG3L2 as a more versatile molecule, able to compensate the absence of its partner paraplegin for essential mitochondrial functions. In particular, complexes differing in subunit composition may operate on different substrates.

Differently from human, yeast and Caenorhabditis elegans, a third homolog, AFG3L1, is present in the mouse. Paraplegin, AFG3L2, and AFG3L1 can form mixed composition-oligomers, but only AFG3L1 and AFG3L2 have the ability to form functional high molecular weight homocomplexes (Koppen et al., 2007). Moreover, even if AFG3L2 and AFG3L1 are ubiquitous proteins, they are differentially expressed in different tissues. In particular, AFG3L2 is largely prevalent in brain, where AFG3L1 is barely expressed. This different expression pattern and the consequent unfeasibility of paraplegin and AFG3L1 to compensate for 
A

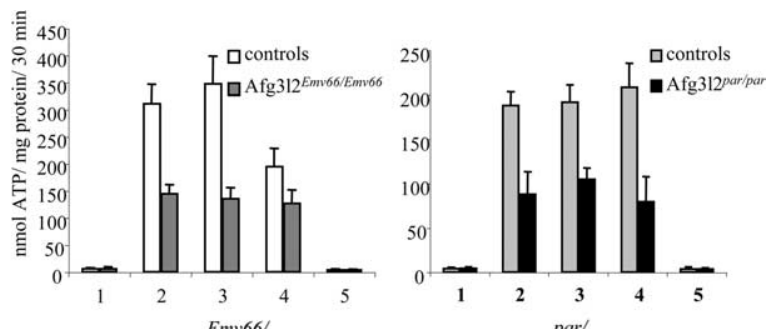

B

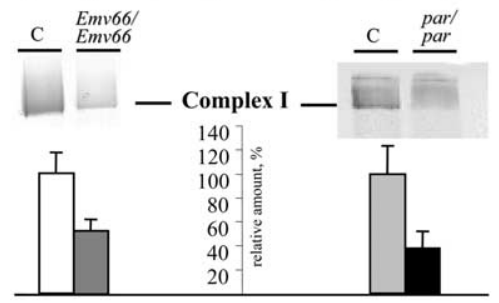

C

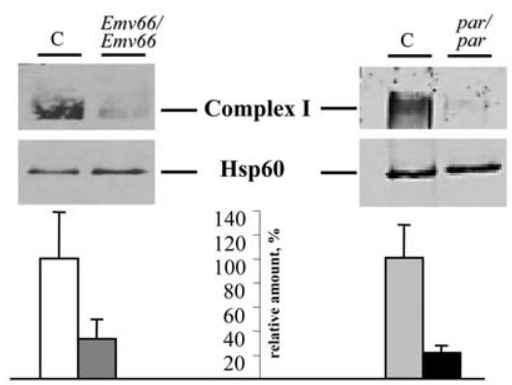

D

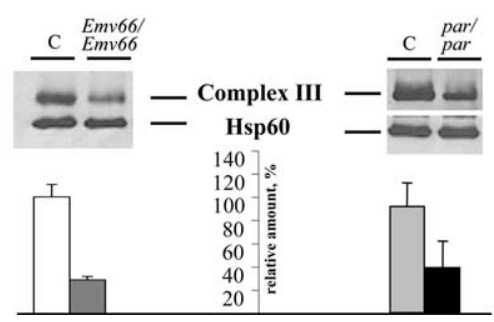

Figure 8. Respiratory chain complexes deficiencies in Afg3/2 mutant mice. A, ATP synthesis rates in mitochondria isolated from brain tissues. 1, No substrates (basal activity); 2 , pyruvate and L-malate (complexes I, II, III, IV, and V); 3, glutamate and L-malate (complexes I, II, III, IV, and V); 4, rotenone and succinate (complexes II, III, IV, and V); 5, antimycin, ascorbate, and TMPD (complexes IV and V). Left graph, Afg3/2 Emv66/Emv66 substrate/t test $p$ value: $2 / 0.008$; $3 / 0.013 ; 4 / 0.044$. Right graph, Afg3/2 $2^{\text {parpar }}$ substrate/t test $p$ value: $2 / 0.0004 ; 3 / 0.0002$; 4/0.0006. B, BN-PAGE and in situ activity staining by nitroblue tetrazolium reduction assay. Comparison are relative to control; Afg312 Emv66/Emv66,$p=0.003 ;$ Afg3/2 ${ }^{\text {par/par }}, p=0.020(t$ test). C, BN-PAGE-Immunoblot analysis of complex I revealed by anti-39 kDa antibody. Afg3/2 ${ }_{\text {Emv66/Emv66 }}, p=0.024 ;$ Afg3/2 $2^{\text {par } / \text { ar }}, p=0.0005$ ( $t$ test). D, BN-PAGE-Immunoblot analysis of complex III revealed by anti-core2 (25.6 kDa subunit) antibody. Afg3/2 ${ }^{\text {Emv66/Emv66 }}, p=$ 0.011 ; Afg $3 / 2^{\text {par/par }}, p=0.004$ ( $t$ test). Activity and protein amount of complex I and III were quantified by densitometric analysis; immunoblotting with $\mathrm{Hsp} 60$ antibody was used to verify equal loading. Error bars represent $\pm S D, n=4$.

AFG3L2 deficiency can explain the more severe neurological phenotype of AFG3L2 mutants.

\section{Mitochondria and axonal maturation}

The presented data show that the mitochondrial damage caused by $m$-AAA deficiency is responsible for the developmental failure observed in Afg3l2 models. Although several lines of evidence indicate that mitochondria play a relevant role in the maintenance of neuronal structure and function (Chang and Reynolds, 2006), this is the first report of a mouse model where axonal development is linked to a mitochondrial protein.

Several hypotheses, not mutually exclusive, can be put forward. As neurons grow, energetic demands extend further from the cell body. Thus, proper function and trafficking of mitochondria are necessary tasks beginning in the earliest moments of neuronal development and becoming increasingly significant as development proceeds.

Another relevant aspect of axonal development is the determination of axonal caliber, which is influenced by intrinsic neuronal factors and extrinsic factors related to myelination (Friede and Samorajski, 1967). In fact, glial cells sense axonal diameter and from this information they determine whether an axon should be myelinated and the size of myelin sheath to synthesize. This correlation is established in the early phases of development and is mediated by axonal Nrg-1-III signaling through ErbB glial receptors (Michailov et al., 2004). Conversely, in the later stages of development, signals from myelinating cells regulate a kinasephosphatase cycle in the axon that maintains the phosphorylation state of neurofilaments. This parameter in turn controls neurofilament density and spacing and thus the axonal caliber (de Waegh et al., 1992). In Afg3l2 mice spinal cord, we observe an impressive reduction of myelinated fibers, suggesting that most of the axons are not able to reach the threshold caliber to be myelinated.

Moreover, the fraction of axons that achieve myelination denotes radial growth impairment both in spinal cord and sciatic nerve. These evidences suggest that mitochondrial $m$-AAA deficiency cause a severe axonal damage in the early phases of development, which in turn results in an inefficient axonal-glial interplay. Many synergistic mechanisms can take part in hampering the cross talk between axons and glial cells. For instance, the aberrant enlarged mitochondria clustered in the cell body observed in Afg3l2 mutant neurons could clog the axonal hillock and/or block molecular motors, thus interfering with proper axonal trafficking. The direct consequence would be the decreased delivery of energy-producing units, mitochondria, and axonal building blocks, such as NF, resulting in energy impairment and a disorganized cytoskeletal network. This hypothesis is supported by the net decrease of NF density and altered axoplasm cytoarchitecture observed in Afg 312 mutant mice. Because NFs are the main determinants of axonal structure and radial growth, this is also consistent with the observed reduced axonal caliber, suggesting that potentially large axons are underdeveloped because they are not supported by adequate NF content. In addition, damaged axons may not respond to myelin signals, leading to inadequate NF phosphorylation. We also cannot exclude a more specific mechanism, where molecules essential for the axon-glia interplay, i.e., Nrg-1-III, are not efficiently transported from the cell body to the axon in mutant mice as a consequence of altered axonal trafficking, thus impairing the axon-glia communication.

Possibly, the aforementioned scenarios simultaneously apply to cause the neuropathological features observed in our Afg 312 mutant mice. Verifying these hypotheses and further dissecting the related molecular mechanisms that link this mitochondrial metalloprotease complexes to both axonal development and neurodegeneration will be goals for future investigations.

\section{References}

Atorino L, Silvestri L, Koppen M, Cassina L, Ballabio A, Marconi R, Langer T, Casari G (2003) Loss of m-AAA protease in mitochondria causes complex I deficiency and increased sensitivity to oxidative stress in hereditary spastic paraplegia. J Cell Biol 163:777-787.

Blondet B, Duxson MJ, Harris AJ, Melki J, Guenet JL, Pincon-Raymond M, Rieger F (1989) Nerve and muscle development in paralyse mutant mice. Dev Biol 132:153-166.

Bross P, Rugarli EI, Casari G, Langer T (2004) Protein quality control in 
mitochondria and neurodegeneration in hereditary spastic paraplegia. Heidelberg: Springer.

Casari G, De Fusco M, Ciarmatori S, Zeviani M, Mora M, Fernandez P, De Michele G, Filla A, Cocozza S, Marconi R, Dürr A, Fontaine B, Ballabio A (1998) Spastic paraplegia and OXPHOS impairment caused by mutations in paraplegin, a nuclear-encoded mitochondrial metalloprotease. Cell 93:973-983.

Chang DT, Reynolds IJ (2006) Mitochondrial trafficking and morphology in healthy and injured neurons. Prog Neurobiol 80:241-268.

de Waegh SM, Lee VM, Brady ST (1992) Local modulation of neurofilament phosphorylation, axonal caliber, and slow axonal transport by myelinating Schwann cells. Cell 68:451-463.

Dubowitz V, ed (1985) Muscle biopsy: a practical approach. London, UK: Balliere-Tindall.

Duchen LW, Gomez S, Guenet J-L, Love S (1983) Paralysè: a new nneurological mutant mouse with progressive atrophy and loss of motor nerve terminals. J Physiol 345:166P.

Ferreirinha F, Quattrini A, Pirozzi M, Valsecchi V, Dina G, Broccoli V, Auricchio A, Piemonte F, Tozzi G, Gaeta L, Casari G, Ballabio A, Rugarli EI (2004) Axonal degeneration in paraplegin-deficient mice is associated with abnormal mitochondria and impairment of axonal transport. J Clin Invest 113:231-242.

Friede RL, Samorajski T (1967) Relation between the number of myelin lamellae and axon circumference in fibers of vagus and sciatic nerves of mice. J Comp Neurol 130:223-231.

Jung C, Higgins CM, Xu Z (2000) Measuring the quantity and activity of mitochondrial electron transport chain complexes in tissues of central nervous system using blue native PAGE. Anal Biochem 286:214-223.

Koppen M, Metodiev MD, Casari G, Rugarli EI, Langer T (2007) Variable and tissue-specific subunit composition of mitochondrial m-AAA protease complexes linked to hereditary spastic paraplegia. Mol Cell Biol $27: 758-767$.
Leonhard K, Stiegler A, Neupert W, Langer T (1999) Chaperone-like activity of the AAA domain of the yeast Ymel AAA protease. Nature 398:348-351.

Michailov GV, Sereda MW, Brinkmann BG, Fischer TM, Haug B, Birchmeier C, Role L, Lai C, Schwab MH, Nave KA (2004) Axonal neuregulin-1 regulates myelin sheath thickness. Science 304:700-703.

Nieuwenhuys R, Ten Donkellar HJ, Nicholson C (1998) The central nervous system of vertebrates. Berlin: Springer.

Nolden M, Ehses S, Koppen M, Bernacchia A, Rugarli EI, Langer T (2005) The m-AAA protease defective in hereditary spastic paraplegia controls ribosome assembly in mitochondria. Cell 123:277-289.

Previtali SC, Quattrini A, Fasolini M, Panzeri MC, Villa A, Filbin MT, Li W, Chiu SY, Messing A, Wrabetz L, Feltri ML (2000) Epitope-tagged P(0) glycoprotein causes Charcot-Marie-Tooth-like neuropathy in transgenic mice. J Cell Biol 151:1035-1046.

Robinson BH (1996) Use of fibroblast and lymphoblast cultures for detection of respiratory chain defects. Methods Enzymol 264:454-464.

Ronner P, Friel E, Czerniawski K, Frankle S (1999) Luminometric assays of ATP, phosphocreatine, and creatine for estimation of free ADP and free AMP. Anal Biochem 275:208-216.

Schagger H, Cramer WA, von Jagow G (1994) Analysis of molecular masses and oligomeric states of protein complexes by blue native electrophoresis and isolation of membrane protein complexes by two-dimensional native electrophoresis. Anal Biochem 217:220-230.

Taylor BA, Frankel WN (1993) A new strain congenic for the Mtv-7/Mls-1 locus of mouse chromosome 1. Immunogenetics 38:235-237.

Taylor BA, Rowe L (1989) A mouse linkage testing stock possessing multiple copies of the endogenous ecotropic murine leukemia virus genome. Genomics 5:221-232.

Trapp BD, Pfeiffer SE, Anitei M, Kidd GJ (2004) Cell biology of myelin assembly. In: Myelin biology and disorders (Lazzarini RA, ed), pp 29-55. London, UK: Elsevier Academic. 